\title{
An Analysis of Emergency Department Visits and the Survival Rate for Colorectal Cancer Patients: A Nationwide Population-based Study
}

\author{
Pei-Ling Tang ${ }^{1-3}$, Hong-Tai Chang ${ }^{4}$, Chin-Chang Cheng ${ }^{5}$, Hung-Chih Chen ${ }^{6}$, \\ Shyh-Ming $\mathrm{Kuo}^{7}$, Kuan-Yin $\mathrm{Hsiao}^{8}$ and Kuo-Chen Chang ${ }^{9}$
}

\begin{abstract}
:
Objective We examined the general characteristics, survival rate, and most common reasons for visiting the emergency department (ED) among colorectal cancer patients in Taiwan. We performed a population-based retrospective study and used data sourced from the National Health Insurance Research Database (NHIRD). Methods The colorectal cancer patient population, their diagnosis, and their medical management at the ED were identified using the Longitudinal Health Insurance Database 2000 (HV) codes and International Classification of Diseases, Ninth Revision, Clinical Modification system. We investigated their reasons for visiting the ED and the medications used there, analyzed their cumulative survival curves using the Kaplan-Meier method, and compared the survival curves with other colorectal cancer patients who had never visited the ED.

Results Between 2000 and 2012, there were 6,532 ED visits by 3,347 colorectal patients, and the number per year increased gradually. The top three most common reasons for visiting ED were ill-defined conditions, abdominal pain, and intestinal obstruction. The overall survival rates of colorectal patients in the ED visit group at 3,5 , and 10 years, were $0.65,0.56$, and 0.47 , respectively, without significant differences from the rates among colorectal cancer patients who did not visit the $\mathrm{ED}(\mathrm{p}=0.2072)$.

Conclusion We described the circumstances of ED visitation by colorectal cancer patients in Taiwan. Health care providers and researchers should pay more attention to improve medical care quality and investigate more details to predict the outcome among colorectal cancer patients.
\end{abstract}

Key words: colorectal cancer, emergency department, survival, Taiwan National Health Insurance Research Database (NHIRD)

(Intern Med 56: 2125-2132, 2017)

(DOI: 10.2169/internalmedicine.7629-16)

\section{Introduction}

Colorectal cancer is the most common cancer among men and the second-most common among women in Taiwan (1). Worldwide, colon and rectal cancers account for approximately 1 million new cancer cases annually, and the number of annual colorectal cancer deaths is approximately half the annual incidence (2). It is widely recognized that environmental factors, especially diet, play a prominent role in the etiology of colorectal cancer (3). The abrupt increase in the incidence of colorectal cancer in Taiwan can be attributed to the consumption of a Western diet high in fat and low in fiber.

\footnotetext{
${ }^{1}$ Research Center of Medical Informatics, Kaohsiung Veterans General Hospital, Taiwan, ${ }^{2}$ College of Nursing, Kaohsiung Medical University, Taiwan, ${ }^{3}$ Department of Nursing, Meiho University, Taiwan, ${ }^{4}$ Department of Surgery, Kaohsiung Veterans General Hospital, Taiwan, ${ }^{5}$ Department of Internal Medicine, Kaohsiung Veterans General Hospital, Taiwan, ${ }^{6}$ Department of Oromaxillofacial Surgery, Kaohsiung Veterans General Hospital, Taiwan, ${ }^{7}$ Department of Biomedical Engineering, I-Shou University, Taiwan, ${ }^{8}$ Radiation Oncology Department, Kaohsiung Municipal United Hospital, Taiwan and ${ }^{9}$ Radiation Oncology Department, Kaohsiung Veterans General Hospital, Taiwan Received: April 25, 2016; Accepted: October 17, 2016; Advance Publication by J-STAGE: August 1, 2017
} Correspondence to Dr. Kuo-Chen Chang, kcchang@vghks.gov.tw 
The standard treatment of colorectal cancer includes surgery, chemotherapy, radiotherapy, and target therapy, depending on the tumor site and stage. The use adjuvant therapy has increased in the past decades. All of the above treatment modalities may induce obvious side effects, prompting patients to visit the emergency department (ED) after their discharge from the hospital. In addition, some end-stage colorectal cancer patients visit the ED for palliative care, such as pain relief. Recent studies have suggested that each cancer patient visits the ED at least twice during the final six months of life (4).

Some researchers have analyzed the whole-population medical utilization of the ED in Taiwan based on the Longitudinal Health Insurance Database (5). However, no analysis has focused on the ED utilization among colorectal cancer patients in Taiwan. In this population-based study, we examined the general characteristics, the most common reasons for visiting the $\mathrm{ED}$, the most commonly used medications, and the survival rate among patients with colorectal cancers visiting the ED from 2000 to 2012 in Taiwan.

\section{Materials and Methods}

\section{Database}

We used data sourced from the Longitudinal Health Insurance Database 2000 (LHID2000), which is derived from the National Health Insurance Bureau (NHIB) records and released by the Taiwan National Health Research Institute (NHRI). Taiwan initiated its National Health Institute (NHI) program in 1995, and coverage has been about $98 \%$ of the entire population since its inception. The LHID2000 comprises registration files and medical claims data for reimbursements of 1,000,000 beneficiaries under the Taiwanese NHI program. The Taiwan NHRI randomly selected these $1,000,000$ beneficiaries from the year 2000 Registry of Beneficiaries ( $n=23.72$ million) of the NHI program. Previous studies have demonstrated the validity of the claims data of the National Health Insurance Research Database (NHIRD) $(6,7)$. To date, hundreds of studies have been published in internationally peer-reviewed journals using data from the NHIRD. In particular, The NHIRD has been used in a previous study to examine the ED utilization of all patients in Taiwan (8). The LHID2000 consists of encrypted de-identified secondary data released to the public for research purposes and was therefore exempted from full review following consultation with the VGHKS's Institutional Review Board.

Patients with colorectal cancer were identified from an analysis of ED encounters from 2000 to 2012, using the Health Insurance Database 2000 (LHID2000), compiled by the Taiwan NHI. Data elements within the LHID2000 include international classification of diseases, ninth revision, clinical modification (ICD-9-CM) codes, patient demographic characteristics, hospital characteristics, and inpatient data for ED admissions.

\section{Sample identification}

The colorectal cancer patient subpopulation was identified using the LHID2000 (HV) codes, which encompass all types of malignancies. The HV is a diagnosis and procedure categorization scheme that collapses ICD-9-CM codes into a smaller number of clinically meaningful categories. To select the study cohort, we identified the 3,347 subjects who had been diagnosed with colorectal cancer (ICD-9-CM codes $153-154,153.5,154.2,154.3,154.8$ then delete) after an ED visit between January 1, 2000, and December 31, 2012.

According to the Centers for Disease Control and Prevention, the International Classification of Diseases, Ninth Revision, Clinical Modification (ICD-9-CM) is the official system of assigning codes to diagnoses and procedures associated with hospital use (including the ED) in Taiwan. Hospital employees typically assign more than one ICD-9-CM code per visit. The ICD-9-CM code listed in diagnosis position one (i.e. listed first) for a visit is considered the primary diagnosis, the ICD-9-CM code in diagnosis position two (i.e. listed second) is considered the secondary diagnosis, and so forth.

\section{Outcome/end-point assessment}

The patient and visit demographic variables for colorectal cancer-related visits from LHID2000 included the visit month, patient sex, age at first visit, teaching level of the hospital, region of hospital, diagnostic position of colorectal, frequency of ED visits, frequency of admittance, and survival. Descriptive statistics, both counts and percentages, were calculated for each of these variables.

The frequencies of the categorized primary ICD-9-CM discharge diagnosis were assessed. The reason prompting an ED visit was defined as the primary ICD-9-CM discharge diagnosis associated with the visit, unless the primary diagnosis was a cancer diagnosis (ICD-9-CM Codes 153-154). In cases where a cancer diagnosis was the primary diagnosis ( $28.62 \%$ of visits), the second listed ICD-9-CM diagnosis was considered to be the reason prompting the ED visit. Cancer diagnoses were excluded from our analysis in order to focus on symptom or complication diagnoses among colorectal cancer patients who visited the ED. The frequencies of categorized medicines were also assessed, and the top 10 most common categories were listed.

\section{Data analyses}

Descriptive statistics are represented as numbers of cases, percentages, and means with the standard deviation (SD) of the ED visitation and admittance frequency. The proportion of visits made by patients with colorectal cancer and the disposition status was determined for the top 10 primary non-cancer diagnoses. The cumulative survival curves were estimated using the Kaplan-Meier method. The comparison of the survival curves was performed by the log-rank test, and a value of $\mathrm{p}<0.05$ (two-sided) was considered significant. All statistical analyses were performed using the Statis- 


\section{ED Visits by colorectal patients in 2000-2012 in Taiwan}

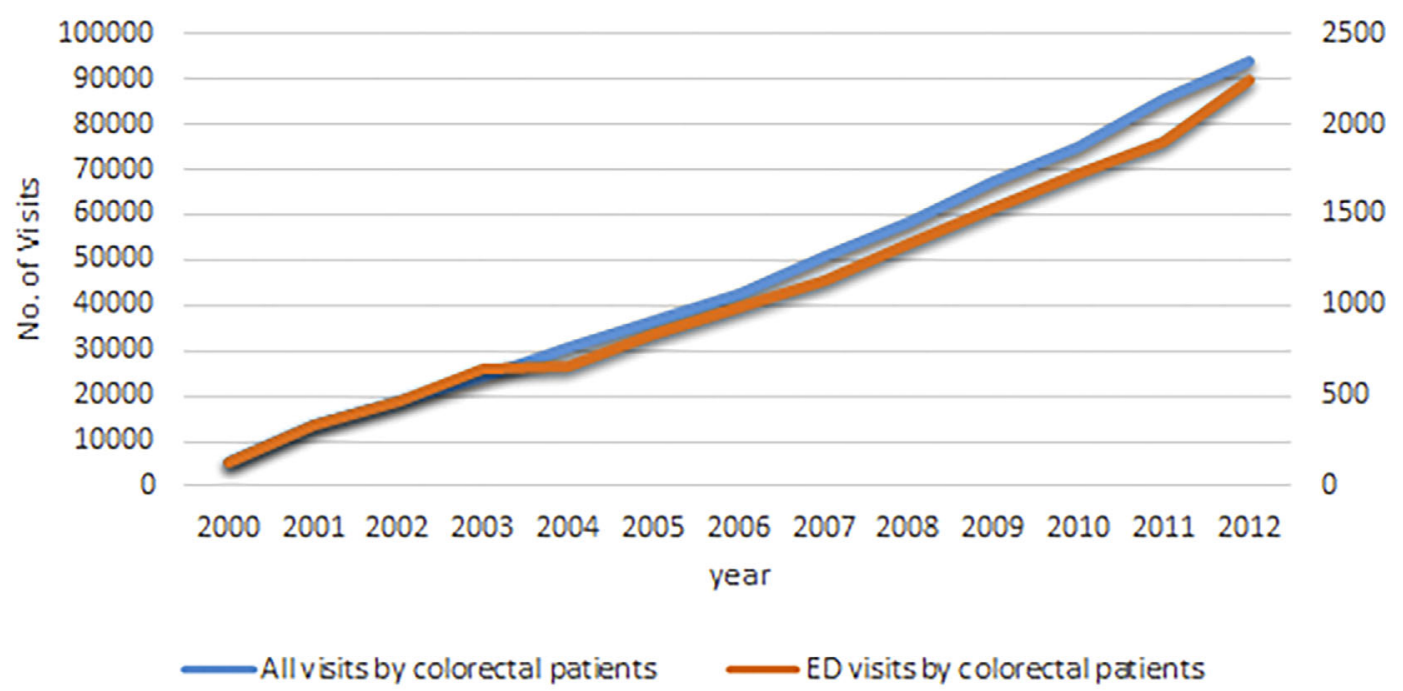

Figure 1. Numbers of all visits (blue line) and emergency department (ED) visits (red line) per year from 2000 to 2012 among colorectal patients.

\section{Percentage of Total ED Visits per Month}

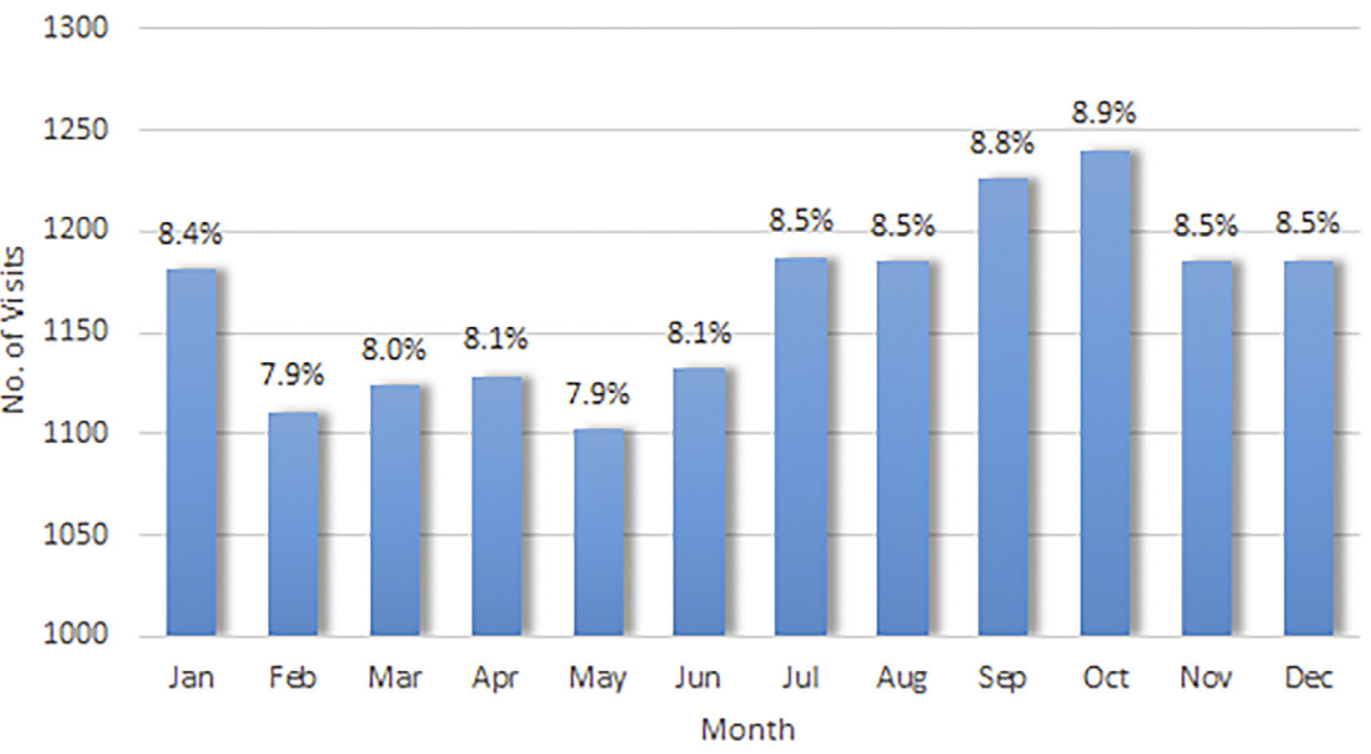

Figure 2. Percentage of emergency department (ED) visits per month from 2000 to 2012 among colorectal patients.

tical Package for SAS software program (SAS System for Windows, version 9.4, SAS Institute Taiwan, Taipei, Taiwan)

\section{Results}

2000-2012 Taiwan ED visits by colorectal cancer patients

Between 2000 to 2012, there were a total of 602,960 colorectal symptom-related visits, which included inpatients, outpatients, and ED services, and the number of visits increased steadily from 5,342 in 2000 to 93,788 in 2012. Among these visits, there were 6,532 ED visits by 3,347 colorectal cancer patients, ranging from 73 visits in 2000 to 997 visits in 2012 (Fig. 1). ED visits were categorized by month of the year (Fig. 2). October (8.9\%) had the highest proportion of visits, and February and May (7.9\%) had the lowest proportion of visits. 


\section{Characteristics of the study population}

The patient characteristics are detailed in Table 1. Over a period of 12 years (2000-2012), 3,347 patients with colorectal cancer visited the ED. Among them, 1,919 (57.33\%) patients were men and $1,428(42.67 \%)$ were women. The mean age of the patients was $66.44 \pm 13.59$ years old. Most of the patients were between 60 and 70 years of age (60-69, $23.9 \%$; age $>70,45.23 \%$ ). Most of the colorectal cancer patients visited the ED at a medical center $(n=1,827,54.59 \%)$ rather than a regional or district hospital $(n=1,520,45.41 \%)$. In addition, most of the patients visited the ED in a densely populated area (Northern, Central, and Southern regions) rather than in a sparsely populated area (Eastern: $n=69$, $2.06 \%$, Islands: $2,0.06 \%$ ). The most common site of colorectal cancer was the colon $(59.28 \%)$, followed by the rectum $(40.22 \%)$ and anus $(0.51 \%)$.

\section{Frequency of ED visits and hospitalizations}

Regarding the proportion of ED visits, 1,006 (30.06\%) patients visited the ED 1 time, $692(20.68 \%)$ patients 2 times, $446(13.33 \%)$ patients 3 times, $272(8.13 \%)$ patients 4 times, $242(7.23 \%)$ patients 5 times, and 689 (20.6\%) patients more than 5 times. The mean number of ED visits was 4.18 \pm 6.85 (Min/Max =1/258). Although 1,573 (47\%) patients didn't require admission, 952 (28.44\%) patients were admitted to the hospital 1 time; 428 (12.79\%) patients 2 times, and $173(5.17 \%)$ patients more than 2 times. The mean number of times admitted was $1.67 \pm 1.62(\mathrm{Min} / \mathrm{Max}=$ 0/17).

\section{Prognosis and survival}

In Table 1, regarding the prognosis of colorectal patients with ED visits, by 2012 , 1,489 (44.49\%) patients had died, and the remaining patients were still alive. We found that the overall survival rates of colorectal cancer patients at 3, 5 , and 10 years decreased over time $(0.65,0.56$, and 0.47 , respectively) in the ED visit group, without significant differences from the rates among colorectal cancer patients who did not visit the ED $(0.68,0.64$, and 0.63 , respectively; $\mathrm{p}=0.2072$; Fig. 3).

\section{Diagnoses and prescription medicines at the ED}

As in Table 2, a rank list of the diagnoses was generated, and the ED visits were categorized based on the top 10 diagnoses (accounted for $92.91 \%$ of all diagnoses) and ranked as other symptoms, signs, and ill-defined conditions (ICD-9CM: 1,701; percentage: $25.59 \%)$; abdominal pain (7,890; $4.67 \%)$; appendicitis and intestinal obstruction without mention of hernia $(540-620 ; 11.43 \%)$; complications of surgical and medical care (996-999, E870-E879, E930-E949; $10.74 \%)$; pyrexia of unknown origin (fever) (7,806; $10.43 \%)$; open wounds and injury to blood vessels (870904; 5.53\%); fractures (800-829, E800-E848; 3.7\%); retention of urine $(7,882 ; 2.83 \%)$; intracranial and internal injuries, including nerves $(850-869,950-957 ; 2.59 \%)$; and con- stipation $(5,640 ; 2.29 \%)$.

Based on the drug prescriptions of the patients with colorectal cancer who visited the ED, 10 categories of drugs (accounting for $95.3 \%$ of all medications) are also listed and ranked in Table 2. Among these 10 drug categories, 4 were gastrointestinal-related medicines $(63.47 \%)$, including agents for the alimentary tract and metabolism (20.61\%), acidrelated disorders $(27.98 \%)$, antiemetics and antinauseants $(12.81 \%)$, and functional gastrointestinal disorders (10.04\%). The remaining categories of drugs were nervous system agents $(7.2 \%)$, antithrombotic agents $(6.59 \%)$, stomatological preparations $(6.59 \%)$, anesthetics $(1.69 \%)$, blood and blood-forming organ agents $(0.91 \%)$, and antiinfectives for systemic use $(0.88 \%)$.

\section{Discussion}

For people with cancer, visiting the ED may be like torture, since waiting for care can be time-consuming and uncomfortable. Having to visit an ED near the end of life is also believed to be indicative of poor-quality care for cancer patients (4). In our study, the number of patients with colorectal cancer who visited the ED rose steadily year over year from 2000 to 2012. Our study showed that a large proportion of colorectal cancer patients visiting the ED were elderly, more than 70 years of age. This finding was compatible with the findings from other researchers that a longer life expectancy has led to more colorectal cancer patients being diagnosed at elderly ages, thereby creating more challenges in subsequent treatment $(9,10)$. Ries et al. (11) found that colorectal cancer patients older than 75 years of age had a lower 5-year relative survival rate than younger patients (58\% vs. 63\%). Another study (12) also noted that the mortality rates of colorectal cancer increased significantly with age, possibly explaining why patients more than 70 years of age account for the majority of total colorectal cancer patients visiting ED.

Regarding the main diagnosis at the ED in our study, approximate $30 \%$ of colorectal cancer patients visited the ED for gastrointestinal-related disease, including abdominal pain, intestinal obstruction, and constipation (Table 2). In our database, $19.62 \%$ of surgeries included a combination of radiotherapy or chemotherapy, and $24.14 \%$ contained chemotherapy alone. The frequently seen postoperative complications include ileus, bleeding, and wound infection. Ileus may be deadly without timely and appropriate management, and its clinical presentation includes abdominal pain and constipation. Therefore, it is reasonable for patients with these clinical symptoms to visit the ED for instant management. Barbera et al. (4) investigated the major reasons for visiting the ED among cancer patients near the end of life, and the most common reason was abdominal pain. In that study, all kinds of cancers were enrolled, and the conclusion was that a high-quality palliative care team could be expected to minimize the number of patients visiting the emergency department. Therefore, more attention should be paid 
Table 1. Demographic Characteristics of Colorectal Patients Presenting to the ED.

\begin{tabular}{|c|c|c|}
\hline Characteristic & No. & $\%$ \\
\hline \multicolumn{3}{|l|}{ Gender } \\
\hline Male & 1,919 & 57.33 \\
\hline Female & 1,428 & 42.67 \\
\hline Age at Diagnosis, years old & \multicolumn{2}{|c|}{ Mean/ SD (66.44/13.59) Min/Max(17.73/106.36) } \\
\hline$<20$ & 1 & 0.03 \\
\hline $20-29$ & 22 & 0.66 \\
\hline $30-39$ & 114 & 3.41 \\
\hline $40-49$ & 293 & 8.75 \\
\hline $50-59$ & 603 & 18.02 \\
\hline $60-69$ & 800 & 23.9 \\
\hline$>70$ & 1,514 & 45.23 \\
\hline \multicolumn{3}{|l|}{ Teaching level of hospitals } \\
\hline Medical center & 1,827 & 54.59 \\
\hline Regional, district hospital & 1,520 & 45.41 \\
\hline \multicolumn{3}{|l|}{ Geographic region } \\
\hline Northern & 1,646 & 49.19 \\
\hline Central & 637 & 19.04 \\
\hline Southern & 992 & 29.65 \\
\hline Eastern & 69 & 2.06 \\
\hline Islands & 2 & 0.06 \\
\hline \multicolumn{3}{|l|}{ Site } \\
\hline Colon & 1,984 & 59.28 \\
\hline Rectal & 1,346 & 40.22 \\
\hline Anal & 17 & 0.51 \\
\hline ED visits(times) & \multicolumn{2}{|c|}{ Mean/ SD (4.181/6.85) Min/Max(1/258) } \\
\hline 1 & 1,006 & 30.06 \\
\hline 2 & 692 & 20.68 \\
\hline 3 & 446 & 13.33 \\
\hline 4 & 272 & 8.13 \\
\hline 5 & 242 & 7.23 \\
\hline$>5$ & 689 & 20.6 \\
\hline Admittances(times) & \multicolumn{2}{|c|}{ Mean/ SD (1.67/1.62) Min/Max(0/17) } \\
\hline 0 & 1,573 & 47 \\
\hline 1 & 952 & 28.44 \\
\hline 2 & 428 & 12.79 \\
\hline 3 & 173 & 5.17 \\
\hline 4 & 100 & 2.99 \\
\hline 5 & 43 & 1.28 \\
\hline$>5$ & 78 & 2.34 \\
\hline \multicolumn{3}{|l|}{ Treatment Modality } \\
\hline Surgery & 306 & 9.14 \\
\hline Surgery \& RT & 48 & 1.43 \\
\hline Surgery \& ChT & 177 & 5.29 \\
\hline Surgery \& ChT \& RT & 177 & 5.29 \\
\hline RT & 126 & 3.76 \\
\hline $\mathrm{RT} \& \mathrm{ChT}$ & 312 & 9.32 \\
\hline ChT & 808 & 24.14 \\
\hline None & 1,393 & 41.62 \\
\hline \multicolumn{3}{|l|}{ Survival } \\
\hline Lived & 1,858 & 55.51 \\
\hline Died & 1,489 & 44.49 \\
\hline
\end{tabular}

ED: emergency department, RT: radiotherapy, ChT: chemotherapy

to post-operative gastrointestinal-related nursing by clinical oncologic caregivers to prevent possible side effects.
Another major reason for colorectal cancer patients visiting the ED was pain, which was evident both in the diagno- 


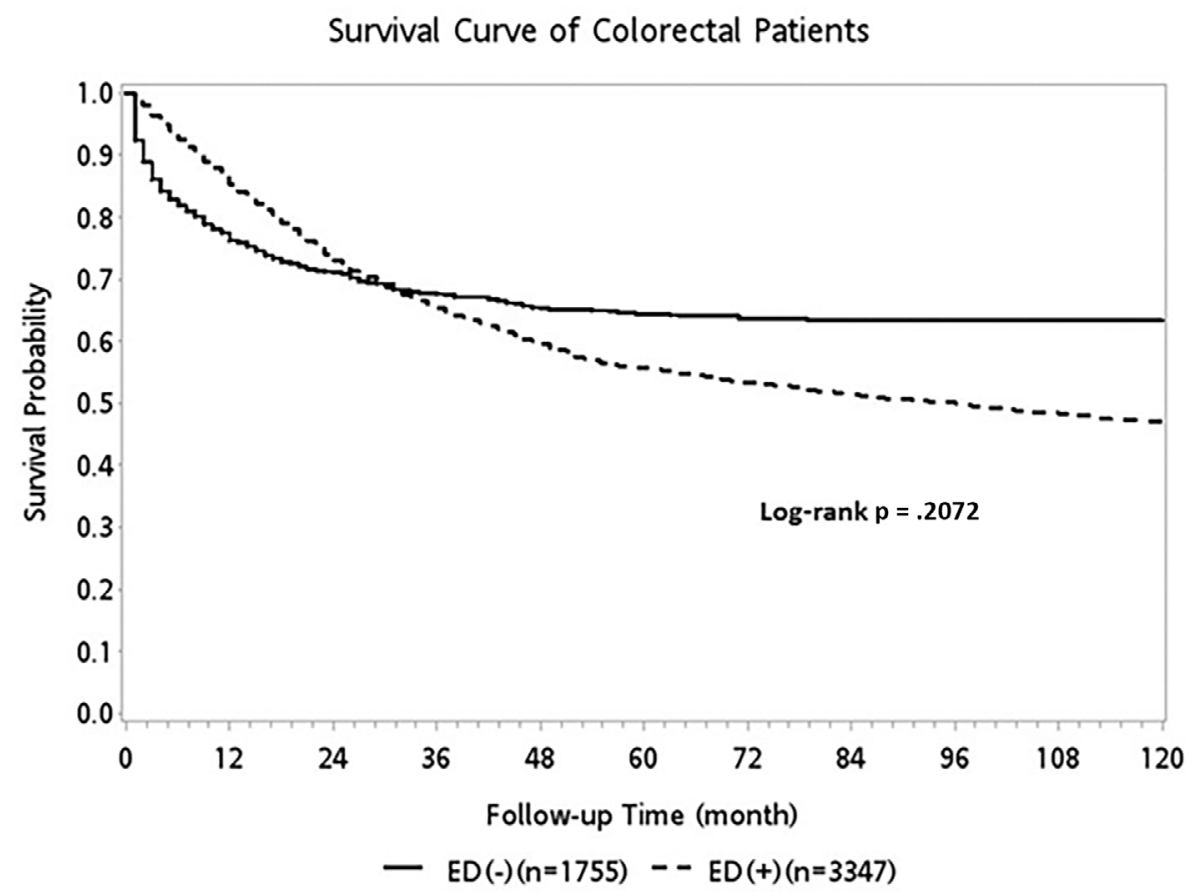

Figure 3. Kaplan-Meier survival curves for colorectal patients with emergency department (ED) visits (dashed line) and colorectal patients without ED visits (solid line).

Table 2. Presenting Diagnoses and Drug Categories Characteristic of Colorectal Patients with ED Visits.

\begin{tabular}{|c|c|c|c|c|c|c|}
\hline \multicolumn{7}{|c|}{ Ranking } \\
\hline & Diagnoses & No. & $\%$ & Drug Categories & No. & $\%$ \\
\hline 1 & $\begin{array}{l}\text { Other symptoms, signs and } \\
\text { ill-defined conditions }\end{array}$ & 1,410 & 25.59 & Drugs for acid related disorders & 17,082 & 27.98 \\
\hline 2 & Abdominal pain & 980 & 17.78 & Alimentary tract and metabolism & 15,869 & 20.61 \\
\hline 3 & $\begin{array}{l}\text { Intestinal obstruction without } \\
\text { mention of hernia }\end{array}$ & 630 & 11.43 & Antiemetics and antinauseants & 7,818 & 12.81 \\
\hline 4 & $\begin{array}{l}\text { Complications of surgical and } \\
\text { medical care }\end{array}$ & 592 & 10.74 & $\begin{array}{l}\text { Drugs for functional } \\
\text { gastrointestinal disorders }\end{array}$ & 6,126 & 10.04 \\
\hline 5 & $\begin{array}{l}\text { Pyrexia of unknown origin } \\
\text { (Fever) }\end{array}$ & 575 & 10.43 & Nervous system & 4,393 & 7.2 \\
\hline 6 & $\begin{array}{l}\text { Open wounds and injury to } \\
\text { blood vessels }\end{array}$ & 305 & 5.53 & Antithrombotic agents & 4,025 & 6.59 \\
\hline 7 & Fractures & 204 & 3.7 & Stomatological preparations & 4,022 & 6.59 \\
\hline 8 & Retention of urine & 156 & 2.83 & Anesthetics & 1,029 & 1.69 \\
\hline 9 & $\begin{array}{l}\text { Intracranial and internal } \\
\text { injuries, including nerves }\end{array}$ & 143 & 2.59 & Blood and blood forming organs & 558 & 0.91 \\
\hline 10 & Constipation & 126 & 2.29 & Antiinfectives for systemic use & 536 & 0.88 \\
\hline
\end{tabular}

ED: emergency department

sis and in the use of anesthetics in this study. Distant bony metastases and local invasion to the sacrum are commonly seen in terminal-stage colorectal cancer; as a result, bone pain is a frequent complaint. Ideally, anesthetics should be prescribed to outpatients once complaints of non-steroidal anti-inflammatory drugs (NSAID)-refractory pain start. This finding suggests that the quality and quantity of cancer pain management should be addressed to a greater degree, both for patients and their clinical caregivers, to avoid visits to the ED.
The current guideline for colorectal cancer is multimodality treatment, including surgery, chemotherapy, target therapy, and radiation therapy (13). Patients may suffer from different kinds and grades of side effects after adjuvant treatment. Neutropenia, diarrhea, and vomiting were the most frequent adverse effects of the commonly prescribed regimen FOLFOX (fluorouracil, leucovorin, and oxaliplatin) (14). Another study (2) noted that $92 \%$ of patients treated with FOLFOX developed peripheral sensory neuropathy of any grade during treatment. In this study, $25.59 \%$ 
of patients presented with discomfort and ill-defined conditions, which could be attributed to combined toxicities of chemotherapy; $10.43 \%$ of patients visited ED for a fever, which could be a sign of chemotherapy-induced neutropenia. Our study also showed that a large proportion $(63.47 \%)$ of colorectal cancer patients were given gastrointestinal-related medications at the ED, which meant that they visited for gastrointestinal-related symptoms, such as diarrhea and vomiting after chemotherapy and radiation therapy. Although surgical techniques and multimodality treatments have improved over the years and decreased rates of postoperative complications have been achieved (15-17), we still noted a continuous increase in the number and proportion of colorectal cancer patients who had visited ED between 2000 and 2012. One of the most likely reasons for this increase is that the incidence of colorectal cancer increased over those years, thereby resulting in greater numbers of patients visiting the ED. Further yearly analyses of the top 10 diagnoses should be performed to clarify why the number of visits to the ED has risen gradually.

A high dose of vitamin $\mathrm{D}$ is known to be able to reduce the incidence of colon cancer (18). On other words, lower serum 25-hydroxyvitamin (a metabolite of vitamin D3) levels may increase the risk of colon cancer. Bischoff-Ferrari et al. (18) further suggested that a high intake of vitamin D leads to a significant reduction in the risk of fracture. People with lower intake and levels of vitamin D are apt to both bone fracture and colon cancer. Moreover, once colon cancer patients develop bony metastases without appropriate treatment, fracture is likely to subsequently occur. These two issues may explain why fracture was ranked as the seventhmost common reason why colorectal cancer patients visited the ED.

The survival rate among colorectal cancer patients in the current study was not significantly affected by visits to the ED. McArdle et al. (19) found that emergency presentation of colorectal cancer was associated with a poor five-year survival rate. Other previous studies (20-22) also highlighted the high postoperative mortality rate and poor survival associated with emergency presentation. Their database population was patients who had suffered from emergent conditions and then underwent resection for colorectal cancer. However, such patients were not included in our study because they did not have a cancer diagnosis when arriving at the ED. Furthermore, it has been recognized that the prognostic factors of colorectal cancer to predict survival consist of cancer stage, tumor grade, Kras gene mutation status, and tumor location. However, no relationship between these factors and the need to visit the ED has been identified.

This is the first longitudinal study to specifically investigate whether or not visiting the ED affects the survival rate of colorectal cancer patients in Taiwan. It also provides insight into the nature of the problem they experienced and the direction of possible interventions. Further evaluations, such as the symptom severity and an annual analysis, should be additionally performed for a more survey of the situation.
Improved medical care quality for colorectal cancer patients should be a goal of researchers and health care providers.

\section{Limitations}

Several limitations associated with the present study warrant mention. First, we did not differentiate the end-stage patients from the general patients with colorectal cancer. As a result, we were unable to determine the actual reason (palliative or otherwise) for visiting the ED. Second, the ICD system was designed for general purposes and was not fit for describing cancer-associated problems (23). To resolve these issues, we ranked the top 10 drugs prescribed to ED patients to attempt to clarify the common reasons and symptoms of colorectal cancer patients visiting ED.

\section{The authors state that they have no Conflict of Interest (COI).}

\section{Acknowledgement}

This study was supported by grants (VGHKS15-EM4-01) from Kaohsiung Veterans General Hospital and the Taiwan Health Promotion Administration. We appreciate being allowed to use the National Health Insurance Research Database provided by the Statistic Center of Department of Health and Welfare. The interpretations and conclusions contained herein do not represent those of the Bureau of Health Promotion, Taiwan, R.O.C.

\section{References}

1. Wang YW. Bureau of Health Promotion, Cancer Registry Annual Report, Taiwan, 2011 [Internet]. [cited 2013 Apr 16]. Available from: http://www.hpa.gov.tw

2. Andre T, Boni C, Navarro M, et al. Improved overall survival with oxaliplatin, fluorouracil, and leucovorin as adjuvant treatment in stage II or III colon cancer in the MOSAIC trial. J Clin Oncol 27: 3109-3116, 2009.

3. Slattery ML, Boucher KM, Caan BJ, et al. Eating patterns and risk of colon cancer. Am J Epidemiol 148: 4-16, 1998.

4. Barbera L, Taylor C, Dudgeon D. Why do patients with cancer visit the emergency department near the end of life? CMAJ 182: 563-568, 2010.

5. Chou HC, Chung CH, Lu P, Kao SY, Lai CH, Chien WC. The injury types and medical expenditure of Taiwanese elderly patients admitted to emergency department in 2007. Taiwan Geriatr Gerontol 6: 15-28, 2011.

6. Hsieh $\mathrm{CY}$, Chen $\mathrm{CH}$, Li CY, et al. Validating the diagnosis of acute ischemic stroke in a National Health Insurance claims database. J Formos Med Assoc 114: 254-259, 2015.

7. Lin HC, Xirasagar S, Chen CH, et al. Physician's case volume of intensive care unit pneumonia admissions and in-hospital mortality. Am J Respir Crit Care Med 177: 989-994, 2008.

8. Nan-Ping Y, Yi-Hui L, Chi-Yu C, et al. Comparisons of medical utilizations and categorical diagnoses of emergency visits between the elderly with catastrophic illness certificates and those without. BMC Health Serv Res 13: 152, 2013.

9. Lemmens VE, Janssen-Heijnen ML, Houterman S, et al. Which comorbid conditions predict complications after surgery for colorectal cancer? World J Surg 31: 192-199, 2007.

10. Ries LAG, Eisner MP, Kosary CL, et al. SEER cancer statistics review 1997 [Internet]. [cited 2001 Apr 1]. Available from: http://see r.cancer.gov

11. Ries LA, Wingo PA, Miller DS, et al. The annual report to the nation on the status of cancer, 1973-1997, with a special section on 
colorectal cancer. Cancer 88: 2398-2424, 2000.

12. Ait Ouakrim D, Pizot C, Boniol M, et al. Trends in colorectal cancer mortality in Europe: retrospective analysis of the WHO mortality database. BMJ 351: h4970, 2015.

13. Andre T, Boni C, Mounedji-Boudiaf L, et al. Oxaliplatin, fluorouracil, and leucovorin as adjuvant treatment for colon cancer. N Engl J Med 350: 2343-2351, 2004.

14. The Clinical Outcomes of Surgical Therapy Study G. A comparison of laparoscopically assisted and open colectomy for colon cancer. N Engl J Med 350: 2050-2059, 2004.

15. Jayne DG, Guillou PJ, Thorpe $\mathrm{H}$, et al. Randomized trial of laparoscopic-assisted resection of colorectal carcinoma: 3-year results of the UK MRC CLASICC Trial Group. J Clin Oncol 25: 3061-3068, 2007.

16. Buunen M, Veldkamp R, Hop WC, et al; Colon Cancer Laparoscopic or Open Resection Study Group. Survival after laparoscopic surgery versus open surgery for colon cancer: long-term outcome of a randomised clinical trial. Lancet Oncol 10: 44-52, 2009.

17. Garland CF, Comstock GW, Garland FC, et al. Serum 25hydroxyvitamin D and colon cancer: eight-year prospective study. Lancet 2: 1176-1178, 1989.

18. Bischoff-Ferrari HA, Willett WC, Orav EJ, et al. A pooled analy- sis of vitamin D dose requirements for fracture prevention. $\mathrm{N}$ Engl J Med 367: 40-49, 2012.

19. McArdle CS, Hole DJ. Emergency presentation of colorectal cancer is associated with poor 5-year survival. Br J Surg 91: 605-609, 2004.

20. Anderson JH, Hole D, McArdle CS. Elective versus emergency surgery for patients with colorectal cancer. Br J Surg 79: 706-709, 1992.

21. Scott NA, Jeacock J, Kingston RD. Risk factors in patients presenting as an emergency with colorectal cancer. Br J Surg 82: 321-323, 1995.

22. Canadian Institute for Health Information (2004-2005). CIHI data quality study of Ontario emergency department visits for fiscal year. Ottawa (ON): 2007 [Internet]. [cited 2015 Jan 4]. Available from: http://secure.cihi.ca/cihiweb/products/vol1_nacrs executive su mmary nov2_2007 .pdf

The Internal Medicine is an Open Access article distributed under the Creative Commons Attribution-NonCommercial-NoDerivatives 4.0 International License. To view the details of this license, please visit (https://creativecommons.org/licenses/ by-nc-nd/4.0/).

(C) 2017 The Japanese Society of Internal Medicine Intern Med 56: 2125-2132, 2017 